Andrés CarriónGarcía $^{1}$ Angela Grisales José Lara

Article info: Received 02.12.2020. Accepted 29.03.2021.

UDC -005.336 .3$ DOI - 10.24874/IJQR15.03-18

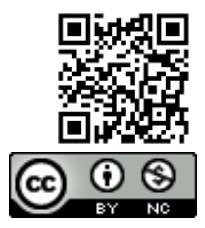

\section{ON THE RELEVANCE OF NDC INDICATOR IN MEASUREMENT SYSTEMS ANALYSIS}

Abstract: A correct performance of measurement systems is a key element in the Quality effort of manufacturing organizations. At least as important as calibration processes, Measurement System Analysis is essential to ensure a solid baseline for decision making. Confirming this relevance, Measurement System Analysis is one of the quality core tools for automotive industry, as ensures the validity of the measurements obtained. In variables measurement, some indicators describe different aspects of the measurement system performance, and simultaneously serve as validation criteria for system acceptance. Probably the most important two indicators are the gage repeatability and reproducibility as a percentage of the total process variation (\%GRR) and the number of distinct categories ( $n d c)$, for which threshold values apply (\%GRR should be less than 10\% and ndc should be greater than 5). In this paper we enhance the fact that these two indicators aren't independent, and the threshold values are incoherent, creating a possible conflict when deciding the acceptance of a measurement system.

Keywords: Measurement systems analysis; GRR; Quality; MSA; Core tools.

\section{Introduction}

The automotive sector has especially highquality requirements, and satisfying these requirements implies the use of advanced tools. A specially valuable set of tools is known as the Core Tools, including under this name Statistical Process Control (SPC), Advance Product Quality Planning (APQP), Production Part Approval Process (PPAP), Failure Mode and Effects Analysis (FMEA) and Measurement System Analysis (MSA).

The last one, MSA, focuses in the fact that quality methodologies require information and data, and these data are usually measured using different gages and measuring instruments. The issue of obtaining reliable information in sectors such as the automotive industry has become highly relevant, given the global spread of suppliers, amongst other reasons. A correct performance of measurement systems is a key element in the Quality effort of manufacturing organizations, at least as important as calibration processes. Measurement System Analysis is essential to ensure a solid baseline for decision making. This fact has even been recognized in international standards, such as ISO 9001:2015, and the more specific IATF 16949:2016 for the aforementioned automotive sector. Authors as Park and Sohn (2005), among many others, enhance the idea of validating measurement systems before use. The way to procure reliable data includes systemic efforts that focus on using metrology laboratories that follow recognized standards and help companies to guarantee metrological traceability via calibration practices.

\footnotetext{
${ }^{1}$ Corresponding author: Andrés Carrión Garcia Email: acarrion@eio.upv.es
} 
However, after having calibrated their measuring equipment, companies still need to check and make sure it performs well under normal working conditions. Although there are different approaches, one of the most relevant is the reference manual developed by AIAG (the Automotive Industry Action Group, which is an association of some of the most important OEMs and suppliers), entitled Measurement System Analysis (MSA), which is now in its fourth edition (AIAG, 2010).

\section{Variation in measurement systems}

There are several variable gage system indicators that evaluate different aspects of gage performance. The best known is Gage Repeatability and Reproducibility (GRR), though it is not the only one. These indicators refer to three aspects of gage performance: variation in position, variation in spread, and sensitivity.

Variations in position affect the mean of the readings obtained in relation to the reference value. Here the most relevant variation is bias, which is the difference between the mean of our readings and the reference value. Stability (the constancy of bias over time) and linearity (degree of dependence of the bias on the magnitude measured) should also be considered.

Variation in spread includes repeatability (usually interpreted as the variation due to the equipment), reproducibility (the variation due to the presence of different appraisers), their combination in GRR (the variation of the measurement system: equipment plus appraisers) and two other types of variations which are similar to stability and linearity, named consistency (constancy of repeatability over time) and uniformity (degree of dependence of the repeatability on the magnitude measured).

The last of the three categories is sensitivity, also known as sensibility or discrimination. This evaluates the ability of the measurement system to distinguish between parts, suitably recognizing the differences between them. In a system with low sensibility, all parts appear to be similar, while a system with good sensibility can recognize the differences in their measurements, constituting what is known as 'categories' of data: groups of measurements which are so similar that they cannot be distinguished by the measurement system. The indicator used here is ndc or number of distinct categories, corresponding to the Signal to Noise Ratio, as commented in Burdick, Morror and Montgomery (2003). The greater the number of categories our measurement system can recognize, the better the system and the more accurate the measurements obtained will be.

\section{Acceptance criteria for a measurement system}

Even though all the variation types mentioned above are important and should be taken into account, the reality of measurement system analysis is that only a few of them are actually used. The most important are bias, GRR and ndc, partly because they are actually relevant and partly because there is a clear criterion for accepting or rejecting the measurement system based on these indicators. Specially in the automotive industry, GRR and ndc are widely used (Barrentine, 2003).

To assess bias, a statistical t-test is performed to test the zero-bias hypothesis, usually at a confidence level of $95 \%$. The system is accepted if the bias is not significantly different from zero, at the desired confidence level.

The GRR criterion consists of calculating the percentage of the total process variation that is covered by the measurement system error (GRR), noted as \%GRR. If this percentage is under $10 \%$, the system is deemed to be acceptable. If it is between $10 \%$ and $30 \%$, the system needs to be improved but may be acceptable for some uses. The system is deemed to be unacceptable if the percentage is over $30 \%$. 
The ndc criterion refers directly to the value of the indicator, and if this is over 5 , the system is deemed to be acceptable.

\section{Acceptance Criteria Discrepancy}

The scenario seems to be clear: if our measurement system fulfils all three criteria it can be considered suitable, depending on what it needs to be used for. Each indicator measures an important aspect of the behavior of the measurement system: position, spread and sensibility (bias, \%GRR and ndc).

The first issue is that there is a direct relationship between two of these indicators, meaning redundancy in the information used (Carrión \& Grisales, 2013). Accordingly, the number of indicators can be reduced, as only two are actually different and independent. The indicators affected are \%GRR and ndc, which are precisely the two that are most widely used to accept measurement systems.

A second problem is that the acceptance criteria established in the fourth edition of the MSA reference manual are inconsistent for these two correlated indicators. The reason could be that when the indicators and criteria were defined, it was assumed that the indicators were independent, and thus criteria could be set independently.

\section{The relationship between \% GRR and ndc}

This relationship is a result of one of the most famous theorems of all the times: the Pythagoras theorem. To understand the question, we must consider the expressions defining GRR and ndc, and review the terminology used.

We will refer to the variation of the parts as $\mathrm{PV}$, when measured without error (i.e.: with a perfect measurement system). We will call total process variation $\mathrm{TV}$, that is the variation observed in measurements as they are taken. This includes variation due to the parts (PV) and variation as an effect of the measurement system. The latter is evaluated through gage repeatability and reproducibility (GRR), which combines the variation caused by the measuring equipment and that caused by the differences between operators. From a statistical point of view, each of these variations is a standard deviation, and can alternatively be noted as $\square \mathrm{P}$ (for PV), $\square \mathrm{T}$ (for TV) and $\square \mathrm{M}$ (for GRR), showing the results of a variance decomposition process.

These three variations, PV, TV and GRR are linked by the expression (AIAG, 2010)

$$
\mathrm{TV}^{2}=\mathrm{PV}^{2}+\mathrm{GRR}^{2}
$$

This expression has a geometric interpretation, as it forms a triangle in which the aforementioned Pythagoras theorem can be applied. In turn, our indicators are defined by (AIAG, 2010):

$$
\begin{aligned}
\% G R R & =\frac{G R R}{T V} \cdot 100 \\
n d c & =1.41 \frac{P V}{G R R}
\end{aligned}
$$

As shown, the elements in both expressions are present in the right triangle that defines the relationship between TV, PV and GRR. By extension, both indicators, \%GRR and ndc, are necessarily linked. Some simple operations using these relations show that the indicators are directly linked by the following expression (Carrión \& Grisales, 2013):

$$
n d c=\sqrt{\frac{20000}{\% G R R^{2}}-2}
$$

This relationship between ndc and \%GRR is presented in Figure 1.

That is, after having computed the \%GRR, we do not really need to compute ndc, as its value is completely defined by the above expression. This is valid both when TV is computed from a representative sample of parts, or when TV is calculated using tolerances and a stated Pp or Ppk value. 
The only difference in this last case is the expression for computing ndc (AIAG, 2010, page 123 ) is:

$$
n d c=1.41 \frac{\sqrt{T V^{2}-G R R^{2}}}{G R R}
$$

These expressions are valid independently of the method used for computing GRR, that is via the mean and range method or using the ANOVA method.
The requirement that \%GRR must be lower than $10 \%$ to constitute an acceptable measurement system, implies having a ndc over 14, far higher than the established criterion of accepting a measurement system with a ndc greater than 5 . In terms of nonacceptability limits, only the criterion of $\%$ GRR over $30 \%$ ties in approximately with that of an ndc lower than 5 (the ndc takes the value 5 when \%GRR is 27 ).

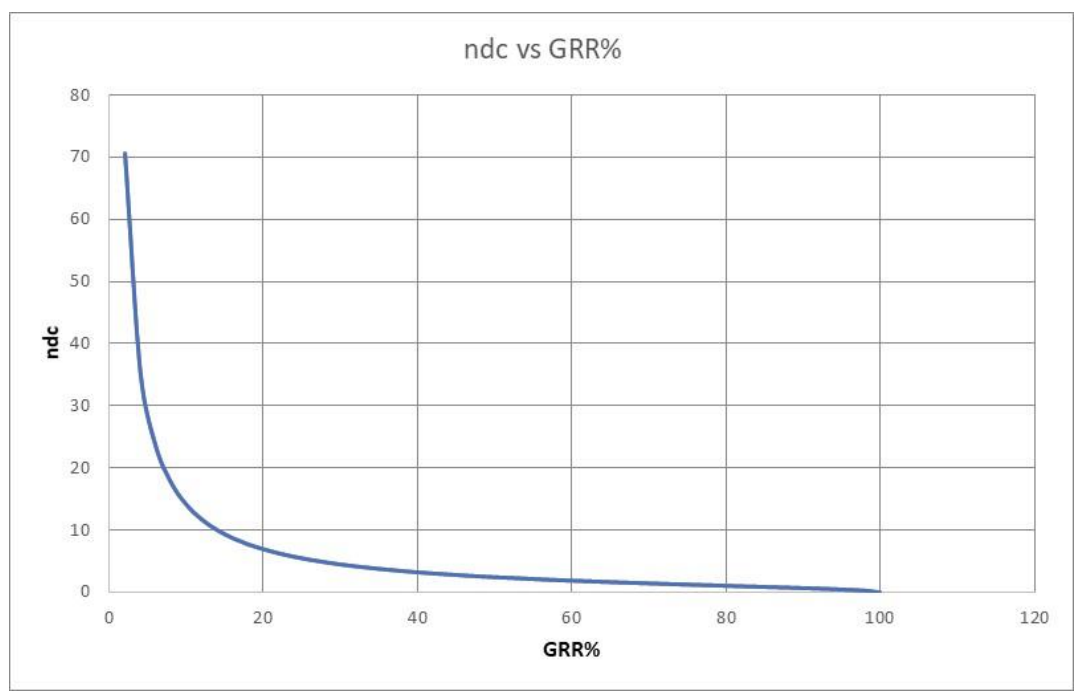

Figure 1. Relationship between ndc and \%GRR.

Figures 2 and 3 show the discrepancy between acceptance/rejection zones for ndc and \%GRR, according to the 4 th edition of the MSA manual (AIAG, 2010).

While the specific acceptance threshold for ndc is 5, the reference manual (AIAG, 2010) states that in some specific cases, measurement systems for ndc over 2 can be useful, but under 2 should be avoided (Barrentine, 2003). This has been included in Figure 3, reinforcing the idea of conflicting criteria.

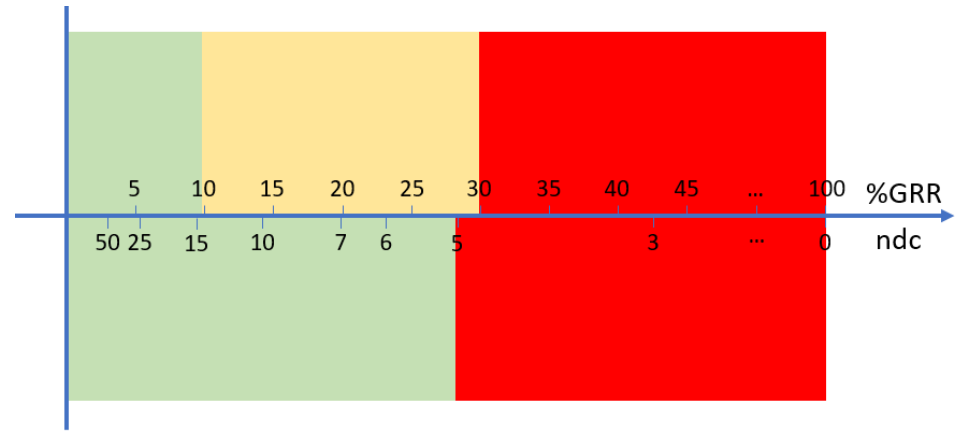

Figure 2. Acceptance/rejection zones according to ndc and \%GRR 


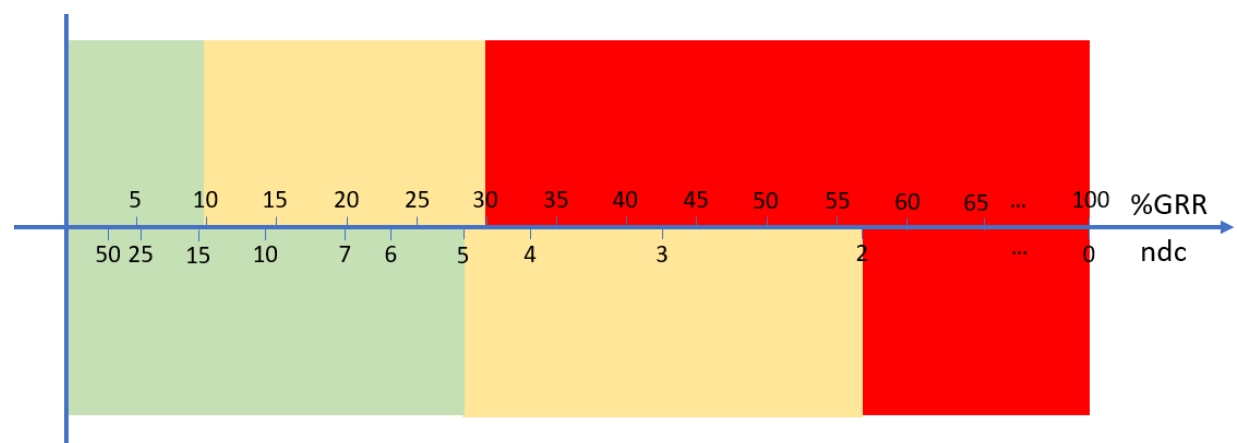

Figure 3. Acceptance/rejection zones according to ndc and \%GRR, with an additional yellow zone for ndc

\section{Conclusions}

After verifying that ndc and \%GRR are linked by the expression shown above, the question of why we need both indicators arises. The original purpose when including ndc was to take into account the relevant item of sensibility, but it has been shown that it is directly linked to \%GRR and, to some extent, we are measuring the same question twice. Thus, ndc adds nothing but confusion, especially when threshold values are considered.

As the more restrictive indicator is \%GRR, this should be prioritized, given that, if the measurement system is acceptable for $\%$ GRR, it will always be acceptable for ndc. So, what should we do with ndc? We can continue to compute it, as required by the reference manual, yet safe in the knowledge that it will never be the first source of problems.

\section{References:}

AIAG (2010). Measurement Systems Analysis reference manual. MSA. Fourth ed. AIAG. Southfield.

Barrentine, L. B. (2003). Concepts for R\&R studies. Second ed., ASQ. Milwaukee.

Burdick, R. K., Borror, C. M., \& Montgomery, D. C. (2003). A Review of Methods for Measurement Systems Capability Analysis. J. Qual. Technol, 35(4), 342-354.

Carrión, A., \& Grisales, A. (2013). Number of distinct data categories and gage repeatability and reproducibility. A double (but single) requirement. Measurement, 46(8), 2514-2518.

Park, T. W., \& Sohn, H.S. (2005). Quality improvement of vehicle drift using statistical six sigma tools. International Journal of Automotive Technology, 6(6), 625-633.

Andrés Carrión-García
Centre for Quality and
Change Management,
Universitat Politecnica de
Valencia,
Valencia,
Spain
acarrion@upv.es

\section{Angela Grisales}

Centre for Quality and

Change Management,

Universitat Politecnica de

Valencia,

Valencia,

Spain

angride1@upv.es

\section{José Lara}

TCM Consulting Group

Valencia,

Spain

joselara@tcmvalencia.es 
QUALITY International Journal for Quality Research 